\title{
The Modified Power Series Inverted Exponentiated Generalized Class of Distributions: Statistical Measures, Model Fit, and Characterization
}

\author{
Clement Boateng Ampadu
}

31 Carrolton Road, Boston, MA 02132-6303, USA

e-mail: drampadu@hotmail.com

\begin{abstract}
Within the master thesis [1, the author considered the following random variable

$$
T=X^{-1}-1
$$

where $X$ follows the Kumaraswamy distribution, and obtains a so-called inverted Kumaraswamy distribution, and studies some properties and applications of this class of distributions in the context of the power series family [2]. Within the paper [3], they introduced the exponentiated generalized class of distributions and obtained some properties with applications. Based on these developments we introduce a class of modified power series inverted exponentiated generalized distributions and obtain some of their properties with applications. Some characterization theorems are also presented. Avenues for further research concludes the paper.
\end{abstract}

\section{Introduction}

The exponentiated class of distributions first appeared in [4, with the following representation for its $\mathrm{CDF}$

Received: July 1, 2020; Accepted: July 23, 2020

2010 Mathematics Subject Classification: 62Exx, 62E10.

Keywords and phrases: exponentiated generalized, power series, characterization theorems, carbon fibers data. 


$$
F(x)^{\beta},
$$

where $F(x)$ is some baseline distribution and $\beta>0$. An extension of this distribution inspired by the Kumaraswamy's double bounded distribution [5] appeared in [6] with the following representation for its $\mathrm{CDF}$

$$
1-\left(1-F(x)^{a}\right)^{b},
$$

where $F(x)$ is some baseline distribution with $a, b>0$. If we exponentiate the above $\mathrm{CDF}$, and take $a=1$, then we get the following CDF introduced in [3]

$$
\left(1-(1-F(x))^{\alpha}\right)^{\beta}
$$

where $\alpha, \beta>0$.

It is well known that the inverse distribution is the distribution of the reciprocal of a random variable [8]. If we consider the random variable $Y=\frac{1}{X}$, where $X$ has CDF $F(x)$, then the reciprocal distribution, $F_{Y}(y)$ has $\mathrm{CDF}$

$$
1-F_{X}\left(\frac{1}{y}\right)
$$

In some recent works, authors have considered the following modification of the transformation $Y=\frac{1}{X}$, that is

$$
Y^{*}=\frac{1}{X}-1
$$

where $X$ has $\mathrm{CDF} F(x)$. In this case, the reciprocal distribution, $F_{Y^{*}}\left(y^{*}\right)$ has $\mathrm{CDF}$

$$
F_{Y^{*}}\left(y^{*}\right)=1-F_{X}\left(\frac{1}{y^{*}+1}\right) .
$$

The power series class of distributions was proposed and studied in [2]. This class of distributions includes binomial, geometric, logarithmic and Poisson distributions as special cases. However, these distributions may not be useful when a random variable takes the value of zero with high probability, that is, zero-inflated. In such 
situations, it is more appropriate to consider the distribution which is truncated at zero.

Power series distributions are usually motivated by the stochastic representations

$$
Z=\min \left(X_{1}, \cdots, X_{N}\right)
$$

if the components are in series, or

$$
Z=\max \left(X_{1}, \cdots, X_{N}\right)
$$

if the components are in parallel, and many lifetime data admit such representations, and the related distributions are very useful in modeling such data. We suppose $X_{1}, \cdots, X_{N}$ are independent and identically distributed random variables from a parent distribution with $\operatorname{PDF} f(x)$ and $\operatorname{CDF} F(x)$, and consider $N$ to be a discrete random variable from a power series distribution (truncated at zero) and whose PDF is given by

$$
P(N=n)=\frac{a_{n} \lambda^{n}}{C(\lambda)}, n=1,2,3, \cdots
$$

where $C(\lambda)=\sum_{n=1}^{\infty} a_{n} \lambda^{n}, a_{n}$ depends on $n$, and $\lambda>0 . C(\lambda)$ is finite, and its first, second, and third derivatives with respect to $\lambda$ are defined and given by $C^{\prime}(\lambda), C^{\prime \prime}(\lambda)$, and $C^{\prime \prime \prime}(\lambda)$, respectively. The table below represents some useful quantities including $a_{n}, C(\lambda), C^{-1}(\lambda), C^{\prime}(\lambda), C^{\prime \prime}(\lambda)$, and $C^{\prime \prime \prime}(\lambda)$, respectively, for the Poisson, geometric, logarithmic and binomial (with $\mathrm{m}$ being the number of replicas) distributions which belong to the power series family of distributions.

Table 1: Useful quantities for some power series distributions.

\begin{tabular}{cccrrrrr}
\hline \hline Distribution & $C(\lambda)$ & \multicolumn{1}{c}{$C^{\prime}(\lambda)$} & $C^{\prime \prime}(\lambda)$ & $C^{\prime \prime \prime}(\lambda)$ & $C^{-1}(\lambda)$ & $a_{n}$ & Parameter Space \\
\hline & & & & & & & \\
Poisson & $e^{\lambda}-1$ & $e^{\lambda}$ & $e^{\lambda}$ & $e^{\lambda}$ & $\log (1+\lambda)$ & $(n !)^{-1}$ & $(0, \infty)$ \\
Geometric & $\lambda(1-\lambda)^{-1}$ & $(1-\lambda)^{-2}$ & $2(1-\lambda)^{-3}$ & $6(1-\lambda)^{-4}$ & $\lambda(1+\lambda)^{-1}$ & 1 & $(0,1)$ \\
Logarithmic & $-\log (1-\lambda)$ & $(1-\lambda)^{-1}$ & $(1-\lambda)^{-2}$ & $2(1-\lambda)^{-3}$ & $1-e^{-\lambda}$ & $n^{-1}$ & $(0,1)$ \\
Binomial & $(1+\lambda)^{m}-1$ & $\frac{m}{(1+\lambda)^{1-m}}$ & $\frac{m(m-1)}{(1+\lambda)^{2-m}}$ & $\frac{m(m-1)(m-2)}{(1+\lambda)^{3-m}}$ & $(\lambda+1)^{\frac{1}{m}}-1$ & $\left(\begin{array}{l}m \\
n\end{array}\right)$ & $(0, \infty)$ \\
\hline
\end{tabular}


If $X_{(1)}=\min \left\{X_{1}, \cdots, X_{N}\right)$, then the conditional CDF of $X_{(1)} \mid N=n$ is given by

$$
F_{X_{(1)} \mid N=n}(x)=1-[1-F(x)]^{n} .
$$

The CDF of the power series class of distributions associated with some parent distribution with PDf $f(x)$ and $\operatorname{CDF} F(x)$ is the marginal CDF of $X_{(1)}$, which is given by

$$
1-\frac{C(\lambda(1-F(x)))}{C(\lambda)}
$$

From [3], the Exponentiated (Standard Uniform) Generalized distribution has cdf

$$
F(x)=\left(1-(1-x)^{\alpha}\right)^{\beta},
$$

where $\alpha, \beta>0$ are two additional shape parameters. Suppose $X$ follows the Exponentiated Standard Uniform Generalized Distribution, and consider the transformation discussed earlier

$$
Y^{*}=X^{-1}-1
$$

By the transformation technique, the cdf of $Y^{*}$ is given by

$$
G\left(y^{*}\right)=1-\left[1-\left(\frac{y^{*}}{y^{*}+1}\right)^{\alpha}\right]^{\beta}
$$

for $\alpha, \beta>0$. The cdf above, represents the cdf of the Inverted Exponentiated (Standard Uniform) Generalized distribution.

Based on the stochastic representation $X_{(1)}=\min \left\{X_{1}, \cdots, X_{N}\right)$ we study some properties and applications of a certain modified power series inverted Exponentiated Generalized distribution (by relaxing the domain space of $y^{*}$ - see new family defined). 


\section{Organization of Manuscript}

This manuscript is organized as follows. In Section 3, we present the PDF and CDF of the new family. In Section 4, we introduce some sub-models of the new family and demonstrate some of their reliablity properties visually. In Section 5, some statistical measures are presented, and they include expansion formulas for the CDF and PDF, quantile function, ordinary moments, Renyi entropy, moment generating function, stochastic ordering, incomplete moments, inequality measures, mean residual life, order statistics, and moments of order statistics. The representation of the distribution as a transformed random variable is also discussed. In Section 6 , estimation of model parameters in the modified Poisson-X family of distributions is discussed. In Section 7 we assess the performance of the maximum likelihood method in estimating model parameters. For this, a sub-model of the broad family is considered, namely, the modified Poission inverted exponentiated generalized distribution (MPIEG), and a monte carlo simulation study is conducted. The result indicates that using the method of maximum likelihood in estimating model parameters is adequate. In Section 8, we show sub-models of the new family are useful in fitting real-life data coming from the chemical/material sciences. In Section 9, we present some characterizations of the Modified Poisson-X distribution. Section 10 concludes the paper, and some directions for further research are proposed.

\section{The New Family Defined}

The PDF of the new family is given by

$$
\frac{\lambda f(x) C^{\prime}(\lambda(1-F(x)))}{C(\lambda)}
$$

where $C$ and $C^{\prime}$ are some useful quantities from Table 1 , and

$$
F(x)=1-\left[1-\left(\frac{x}{x+1}\right)^{\alpha}\right]^{\beta}
$$


for $\alpha, \beta, x>0$ with $f(x)=\frac{\partial F(x)}{\partial x}$. The CDF of the new family is given by

$$
1-\frac{C(\lambda(1-F(x)))}{C(\lambda)}
$$

where $C$ is some useful quantity from Table 1 , and $F(x)$ is as given above. The parameter space for $\lambda$ is $(-\infty, 0) \cup(0, \infty)$.

\section{Some Sub-Models}

We introduce four sub-models and visualize some of their basic reliability properties. The reliability properties include survival function (SF), hazard rate function(HF), and the cumulative hazard rate function $(\mathrm{CHF})$.

\subsection{Modified Poisson Inverted Exponentiated Generalized Distribution}

The PDF of the Modified Poisson Inverted Exponentiated Generalized distribution is given by

$$
P(x ; \alpha, \beta, \lambda)=\frac{\alpha \beta \lambda x^{\alpha-1}(x+1)^{-\alpha-1}\left(1-\left(\frac{x}{x+1}\right)^{\alpha}\right)^{\beta-1} e^{\lambda\left(1-\left(\frac{x}{x+1}\right)^{\alpha}\right)^{\beta}}}{e^{\lambda}-1}
$$

and the CDF is given by

$$
p(x ; \alpha, \beta, \lambda)=\frac{e^{\lambda}-e^{\lambda\left(1-\left(\frac{x}{x+1}\right)^{\alpha}\right)^{\beta}}}{e^{\lambda}-1} .
$$

The survival function, hazard rate function, and the cumulative hazard rate function, respectively, are given by

$$
\begin{aligned}
& S(x ; \alpha, \beta, \lambda)=1-P(x ; \alpha, \beta, \lambda) \\
& H(x ; \alpha, \beta, \lambda)=\frac{p(x ; \alpha, \beta, \lambda)}{1-P(x ; \alpha, \beta, \lambda)}
\end{aligned}
$$




$$
C H F(x ; \alpha, \beta, \lambda)=-\log (S(x ; \alpha, \beta, \lambda)) .
$$

Notation 4.1. We write

$$
W^{*} \sim \operatorname{MPIEG}(\alpha, \beta, \lambda)
$$

if $W^{*}$ is a Modified Poisson Inverted Exponentiated Generalized random variable.
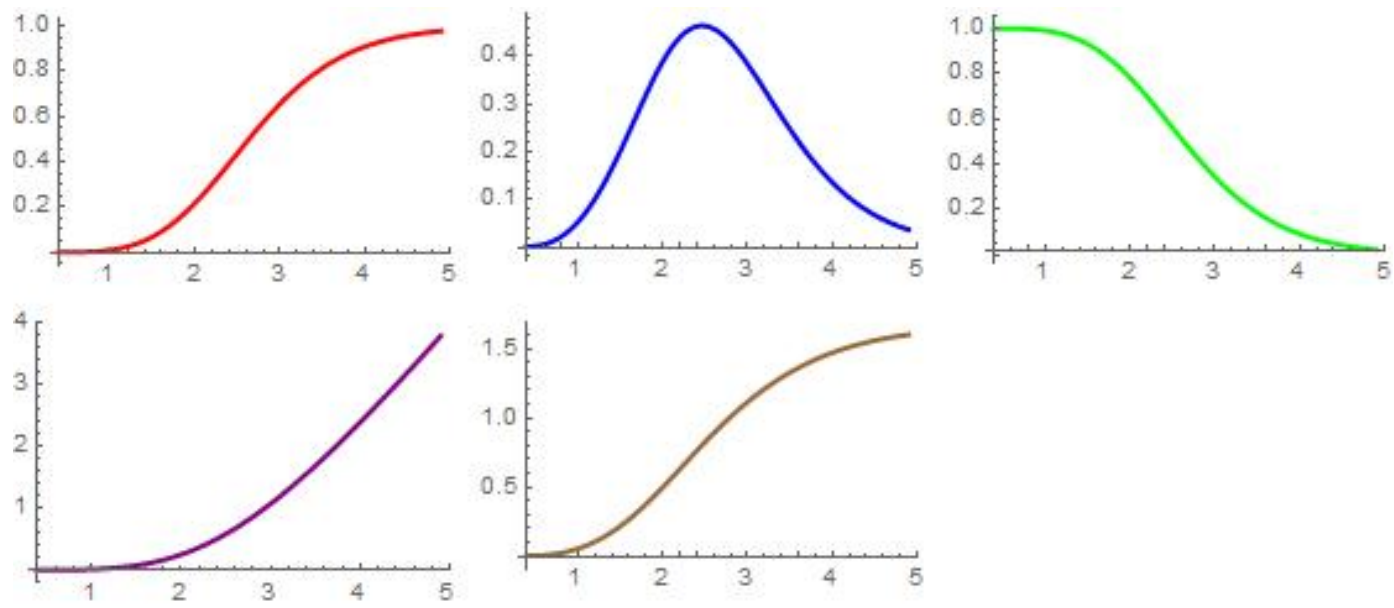

Figure 1: The CDF(red), $\mathrm{PDF}$ (blue), $\mathrm{SF}$ (green), $\mathrm{CHF}$ (purple), and the HF(brown) of MPIEG(8.3, 20.5, -2.9).

\subsection{Modified Geometric Inverted Exponentiated Generalized Distribution}

The PDF of the Modified Geometric Inverted Exponentiated Generalized distribution is given by 


$$
g(x ; \alpha, \beta, \lambda)=-\frac{\alpha \beta(\lambda-1) x^{\alpha-1}(x+1)^{-\alpha-1}\left(1-\left(\frac{x}{x+1}\right)^{\alpha}\right)^{\beta-1}}{\left(\lambda\left(1-\left(\frac{x}{x+1}\right)^{\alpha}\right)^{\beta}-1\right)^{2}}
$$

and the CDF is given by

$$
G(x ; \alpha, \beta, \lambda)=\frac{1}{\lambda+\frac{\lambda-1}{\left(1-\left(\frac{x}{x+1}\right)^{\alpha}\right)^{\beta}-1}} .
$$

The survival function, hazard rate function, and the cumulative hazard rate function, respectively, are given by

$$
\begin{gathered}
S(x ; \alpha, \beta, \lambda)=1-G(x ; \alpha, \beta, \lambda) \\
H(x ; \alpha, \beta, \lambda)=\frac{g(x ; \alpha, \beta, \lambda)}{1-G(x ; \alpha, \beta, \lambda)} \\
C H F(x ; \alpha, \beta, \lambda)=-\log (S(x ; \alpha, \beta, \lambda)) .
\end{gathered}
$$

Notation 4.2. We write

$$
W^{* *} \sim \operatorname{MGIEG}(\alpha, \beta, \lambda)
$$

if $W^{* *}$ is a Modified Geometric Inverted Exponentiated Generalized random variable. 

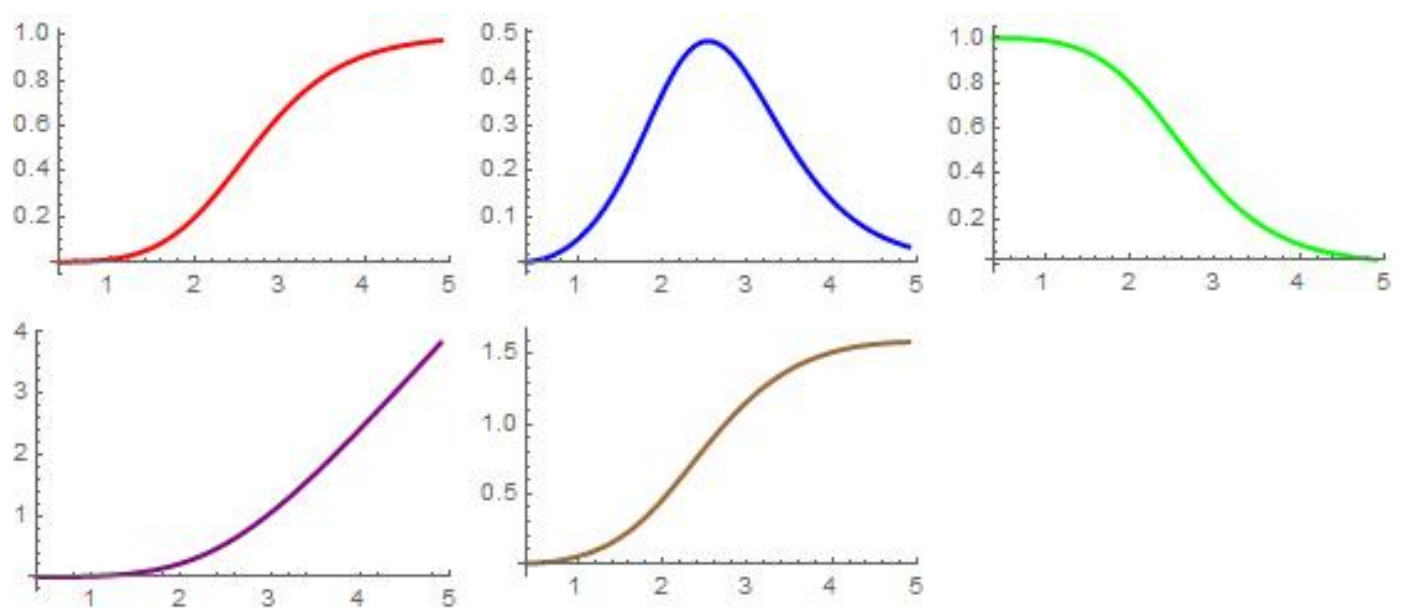

Figure 2: The CDF(red), PDF(blue), SF(green), CHF(purple), and the HF(brown) of MGIEG(5.2, 15.5, -30.3).

\subsection{Modified Binomial Inverted Exponentiated Generalized Distribution}

Here we assume the number of replicas is given by $m=2$. Thus, the PDF of the Modified Binomial Inverted Exponentiated Generalized distribution is given by

$$
b(x ; \alpha, \beta, \lambda)=\frac{2 \alpha \beta x^{\alpha-1}(x+1)^{-\alpha-1}\left(1-\left(\frac{x}{x+1}\right)^{\alpha}\right)^{\beta-1}\left(\lambda\left(1-\left(\frac{x}{x+1}\right)^{\alpha}\right)^{\beta}+1\right)}{\lambda+2}
$$

and the CDF is given by

$$
B(x ; \alpha, \beta, \lambda)=1-\frac{\left(\lambda\left(1-\left(\frac{x}{x+1}\right)^{\alpha}\right)^{\beta}+1\right)^{2}-1}{\lambda(\lambda+2)} .
$$

The survival function, hazard rate function, and the cumulative hazard rate function, respectively, are given by 


$$
\begin{gathered}
S(x ; \alpha, \beta, \lambda)=1-B(x ; \alpha, \beta, \lambda) \\
H(x ; \alpha, \beta, \lambda)=\frac{b(x ; \alpha, \beta, \lambda)}{1-B(x ; \alpha, \beta, \lambda)} \\
C H F(x ; \alpha, \beta, \lambda)=-\log (S(x ; \alpha, \beta, \lambda)) .
\end{gathered}
$$

Notation 4.3. We write

$$
W^{* * *} \sim \operatorname{MBIEG}(\alpha, \beta, \lambda)
$$

if $W^{* * *}$ is a Modified Binomial Inverted Exponentiated Generalized random variable.
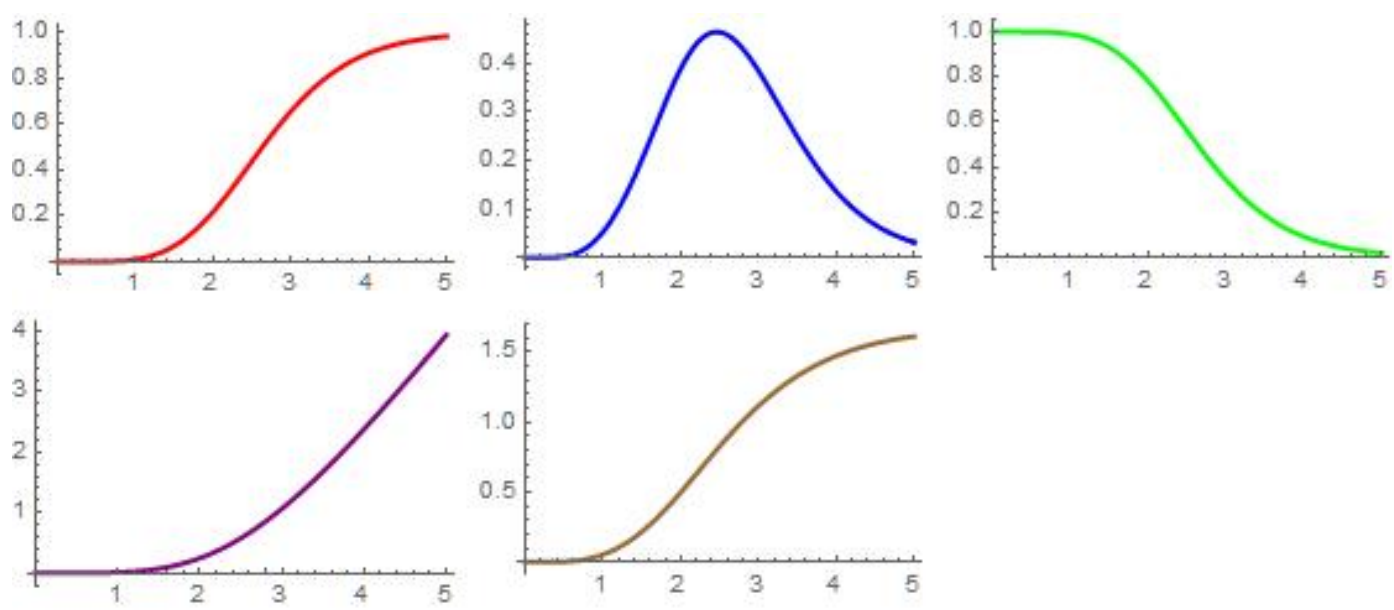

Figure 3: The CDF(red), PDF(blue), SF(green), CHF(purple), and the HF(brown) of MBIEG(10.2,25.8,-0.7). 


\subsection{Modified Logarithmic Inverted Exponentiated Generalized Distribution}

The PDF of the Modified Logarithmic Inverted Exponentiated Generalized distribution is given by

$$
l(x ; \alpha, \beta, \lambda)=\frac{\alpha \beta \lambda x^{\alpha-1}(x+1)^{-\alpha-1}\left(1-\left(\frac{x}{x+1}\right)^{\alpha}\right)^{\beta-1}}{\log (1-\lambda)\left(1-\lambda\left(1-\left(\frac{x}{x+1}\right)^{\alpha}\right)^{\beta}\right)}
$$

and the CDF is given by

$$
L(x ; \alpha, \beta, \lambda)=1-\frac{\log \left(1-\lambda\left(1-\left(\frac{x}{x+1}\right)^{\alpha}\right)^{\beta}\right)}{\log (1-\lambda)} .
$$

The survival function, hazard rate function, and the cumulative hazard rate function, respectively, are given by

$$
\begin{gathered}
S(x ; \alpha, \beta, \lambda)=1-L(x ; \alpha, \beta, \lambda) \\
H(x ; \alpha, \beta, \lambda)=\frac{l(x ; \alpha, \beta, \lambda)}{1-L(x ; \alpha, \beta, \lambda)} \\
C H F(x ; \alpha, \beta, \lambda)=-\log (S(x ; \alpha, \beta, \lambda)) .
\end{gathered}
$$

Notation 4.4. We write

$$
W^{* * * *} \sim \operatorname{MLIEG}(\alpha, \beta, \lambda)
$$

if $W^{* * * *}$ is a Modified Logarithmic Inverted Exponentiated Generalized random variable. 

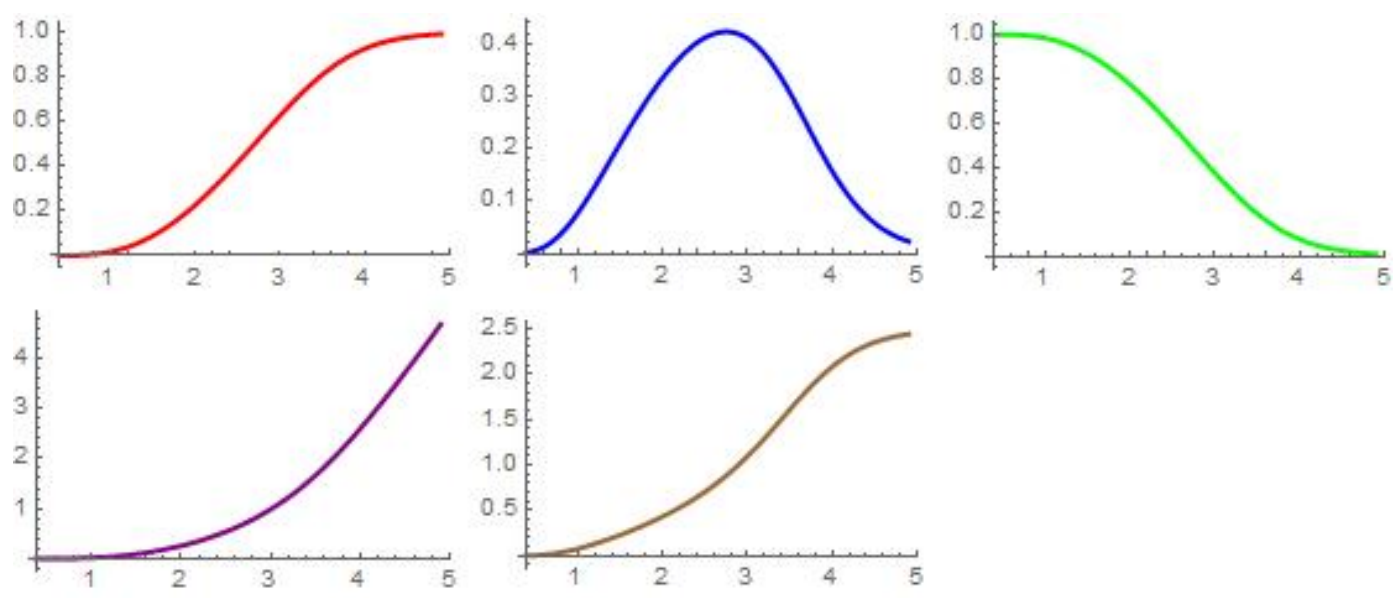

Figure 4: The CDF(red), PDF(blue), SF(green), CHF(purple), and the HF(brown) of MLIEG(8.9, 34.1, -53.8).

\section{$5 \quad$ Some Statistical Measures}

Here we obtain some properties for the Modified Poisson-X distribution, that is, we consider

$$
1-\frac{C(\lambda(1-F(x)))}{C(\lambda)}
$$

with $C(\lambda)=e^{\lambda}-1$.

Explicitly, the CDF can be written as

$$
1-\frac{e^{\lambda(1-F(x))}-1}{e^{\lambda}-1}
$$

where $\lambda \in(-\infty, 0) \cup(0, \infty)$ and $F(x)$ is some baseline CDF. 


\subsection{CDF Power Series}

Theorem 5.1. The CDF of the Modified Poisson-X distribution admit the following representation

$$
\frac{e^{\lambda}-\sum_{k=0}^{\infty} \sum_{q=0}^{k} \frac{\lambda^{k}}{k !}\left(\begin{array}{l}
k \\
q
\end{array}\right)(-1)^{q} F(x)^{q}}{e^{\lambda}-1},
$$

where $\lambda \in(-\infty, 0) \cup(0, \infty)$ and $F(x)$ is some baseline $C D F$.

\subsection{PDF Power Series}

By differentiation, the PDF of the Modified Poisson-X family is given by

$$
\frac{\lambda}{e^{\lambda}-1} f(x) e^{\lambda(1-F(x))}
$$

Theorem 5.2. The PDF of the Modified Poisson-X distribution admit the following representation

$$
\frac{\lambda^{k+1}}{\left(e^{\lambda}-1\right) k !} \sum_{k=0}^{\infty} \sum_{q=0}^{k}(-1)^{q}\left(\begin{array}{l}
k \\
q
\end{array}\right) f(x) F(x)^{q},
$$

where $\lambda \in(-\infty, 0) \cup(0, \infty)$, and the random variable $X$ has PDF $f(x)$ and $C D F$ $F(x)$.

\subsection{Transformation of Random Variables}

Theorem 5.3. Let $U$ be a random variable uniform on $[0,1)$. Then the following random variable follows the Modified Poisson-X class of distributions

$$
X=Q_{F}\left[\frac{\lambda-\log \left(\left(e^{\lambda}-1\right)(1-U)\right.}{\lambda}\right],
$$

where $Q_{F}=F^{-1}(\cdot)$ is the quantile function of the distribution with $C D F F$, and $\lambda \in(-\infty, 0) \cup(0, \infty)$. 


\subsection{Quantile Function}

Theorem 5.4. The quantile function of the Modified Poisson-X class of distributions is given by

$$
Q_{F}\left[\frac{\lambda-\log \left(\left(e^{\lambda}-1\right)(1-p)\right.}{\lambda}\right],
$$

where $Q_{F}=F^{-1}(\cdot)$ is the quantile function of the distribution with $C D F F, \lambda \in$ $(-\infty, 0) \cup(0, \infty)$, and $0<p<1$.

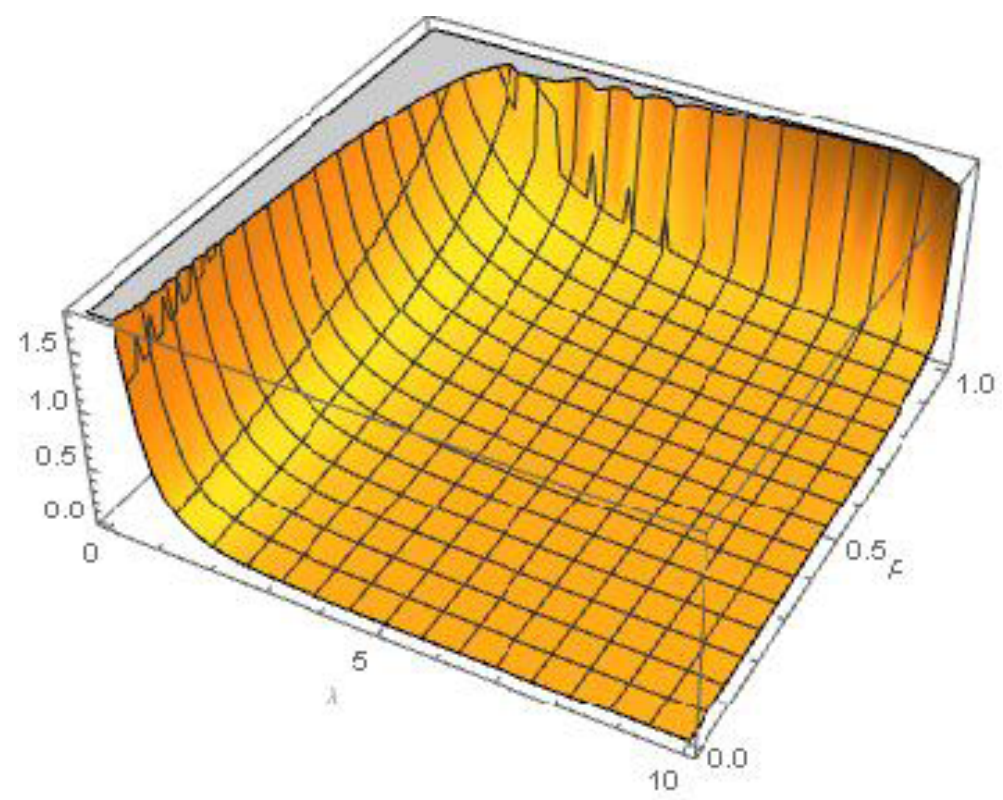

Figure 5: The quantile function of Modified Poisson-Standard Uniform for $0<\lambda<$ 10 and $0<p<10$.

\subsection{Ordinary Moments}

If $X$ is standard uniform, then the PDF of the Modified Poisson-Standard Uniform distribution is given by 


$$
\frac{\lambda}{e^{\lambda}-1} e^{\lambda(1-x)}
$$

for $x \in[0,1]$ and $\lambda \in(-\infty, 0) \cup(0, \infty)$. Thus we have the following

Theorem 5.5. The ordinary moments of the Modified Poisson-Standard Uniform class of distributions are given by

$$
\mu_{r}^{\prime}=\frac{\lambda}{e^{\lambda}-1} e^{\lambda} \lambda^{-r-1}(\Gamma(r+1)-\Gamma(r+1, \lambda))
$$

where $r \in \mathbb{N}, \lambda \in(-\infty, 0) \cup(0, \infty), \Gamma(\cdot)$ is the Euler Gamma function, and $\Gamma(\cdot, \cdot)$ is the incomplete gamma function.

Remark 5.6. By definition,

$$
\Gamma(z)=\int_{0}^{\infty} t^{z-1} e^{-t} d t
$$

and

$$
\Gamma(a, z)=\int_{z}^{\infty} t^{a-1} e^{-t} d t
$$

Table 2: Some values of the ordinary moments of the Modified Poisson-Standard Uniform distribution for some choice parameter $\lambda$.

\begin{tabular}{ccc}
\hline$\mu_{r}^{\prime}$ & $\lambda=0.5$ & $\lambda=2.5$ \\
\hline$\mu_{1}^{\prime}$ & 0.458506 & 0.310575 \\
$\mu_{2}^{\prime}$ & 0.29253 & 0.159034 \\
$\mu_{3}^{\prime}$ & 0.213683 & 0.101415 \\
$\mu_{4}^{\prime}$ & 0.167973 & 0.0728392 \\
$\mu_{5}^{\prime}$ & 0.13824 & 0.056253 \\
\hline
\end{tabular}




\subsection{Renyi Entropy}

Let $X$ be a random variable with $\operatorname{PDF} f(x)$. By definition, the Renyi entropy [9] is defined as

$$
I_{R}(\delta)=\frac{1}{1-\delta} \log \left(\int_{-\infty}^{\infty} f^{\delta}(x) d x\right)
$$

where $\delta>0$, and $\delta \neq 1$. The main result of this section is the following whose proof is omitted.

Theorem 5.7. The Renyi entropy of the Modified Poisson-X family of distributions can be expressed as

$$
I_{R}(\delta)=\frac{1}{1-\delta} \log \left[\left(\frac{\lambda}{e^{\lambda}-1}\right)^{\delta} \sum_{k=0}^{\infty} \sum_{m=0}^{k} \omega_{k, m} \int_{-\infty}^{\infty} f(x)^{\delta} F(x)^{m} d x\right],
$$

where the random variable $X$ has $C D F F(x)$ and $P D F f(x), 1 \neq \delta>0, \lambda \in$ $(-\infty, 0) \cup(0, \infty)$, and

$$
\omega_{k, m}=\left(\begin{array}{c}
k \\
m
\end{array}\right) \frac{\delta^{k} \lambda^{k}(-1)^{m}}{k !}
$$

Table 3: The Renyi entropy of the Modified Poisson-Standard Uniform distribution for some choice parameter $\lambda$.

\begin{tabular}{ccc}
\hline$I_{R}(\delta)$ & $\lambda=0.5$ & $\lambda=2.5$ \\
\hline$I_{R}(0.2)$ & -0.00207797 & -0.0490209 \\
$I_{R}(0.7)$ & -0.00725856 & -0.163813 \\
$I_{R}(1.3)$ & -0.0134301 & -0.281075 \\
$I_{R}(1.5)$ & -0.0154721 & -0.314715 \\
$I_{R}(2.7)$ & -0.0274999 & -0.467369 \\
\hline
\end{tabular}




\subsection{Moment Generating Function}

Given a random variable $X$, one defines the moment generating function as

$$
M_{X}(z)=E\left[e^{z X}\right]
$$

where $E[\cdot]$ is an expectation. Now using the series expansion for $e^{z X}$, one can write

$$
M_{X}(z)=\sum_{r=0}^{\infty} \frac{z^{r} \mu_{r}^{\prime}}{r !}
$$

where $\mu_{r}^{\prime}$ is the $r$ th non-central moment of the random variable $X$. Thus from Theorem 5.5, the following is an immediate consequence

Theorem 5.8. The moment generating function of the Modified Poisson-Standard Uniform class of distributions is given by

$$
M_{X}(z)=\sum_{r=0}^{\infty} \frac{z^{r} \lambda}{r !\left(e^{\lambda}-1\right)} e^{\lambda} \lambda^{-r-1}(\Gamma(r+1)-\Gamma(r+1, \lambda)),
$$

where $\lambda \in(-\infty, 0) \cup(0, \infty), \Gamma(\cdot)$ is the Euler Gamma function, and $\Gamma(\cdot, \cdot)$ is the incomplete gamma function.

\subsection{Stochastic Ordering}

Stochastic ordering is a common concept to show the ordering mechanism in lifetime distributions [10]. A random variable $X$ is said to be stochastically smaller than the random variable $Y$ in the likelihood ratio order $\left(X \leq_{l r} Y\right)$ if the ratio of the associated PDFs given by $\frac{f_{X}(x)}{f_{Y}(x)}$ decreases in $x$. The main result is the following whose proof we omit.

Theorem 5.9. Let $X$ follow the Modified Poisson Standard Uniform distribution with parameter $\lambda_{1}$, and let $Y$ follow Poisson Standard Uniform distribution with parameter $\lambda_{2}$. Let $f_{X}(x)$ and $f_{Y}(y)$ denote the PDF's of $X$ and $Y$, respectively. If $\lambda_{2}<\lambda_{1}$, then $X \leq_{l r} Y$. 


\subsection{Incomplete Moments}

The $r$ th incomplete moment is given by $M_{r}(y)=\int_{-\infty}^{y} x^{r} f(x) d x$. For the Modified Poisson-X family of distributions, we have the following

Theorem 5.10. The rth incomplete moment for the Modified Poisson-X family of distributions, can be expressed as

$$
M_{r}(y)=\frac{\lambda^{k+1}}{\left(e^{\lambda}-1\right) k !} \sum_{k=0}^{\infty} \sum_{q=0}^{k}(-1)^{q}\left(\begin{array}{l}
k \\
q
\end{array}\right) \int_{-\infty}^{y} x^{r} f(x) F(x)^{q} d x,
$$

where $\lambda \in(-\infty, 0) \cup(0, \infty)$, and the random variable $X$ has $P D F f(x)$ and $C D F$ $F(x)$.

\subsection{Inequality Measures}

The Lorenz curve, $L_{F}(y)$ for incomplete moments is defined as $L_{F}(y)=$ $\frac{1}{\mu} \int_{-\infty}^{y} x f(x) d x$. For the Modified Poisson-X family of distributions, we have the following

Theorem 5.11. The Lorenz curve for the Modified Poisson-X family of distributions is given by

$$
L_{F}(y)=\frac{\lambda^{k+1}}{\mu\left(e^{\lambda}-1\right) k !} \sum_{k=0}^{\infty} \sum_{q=0}^{k}(-1)^{q}\left(\begin{array}{l}
k \\
q
\end{array}\right) \int_{-\infty}^{y} x f(x) F(x)^{q} d x,
$$

where $\lambda \in(-\infty, 0) \cup(0, \infty)$, the random variable $X$ has PDF $f(x)$ and $C D F F(x)$, and $\mu$ is the mean of the Poisson- $X$ family.

The Bonferroni curve, $B_{F}(y)$ is defined as $B_{F}(y)=\frac{L_{F}(y)}{F(y)}$. For the Modified Poisson-X family of distributions, we have the following

Theorem 5.12. The Bonferroni curve of the Modified Poisson-X family can be expressed as

$$
B_{F}(y)=\frac{\frac{\lambda^{k+1}}{\mu\left(e^{\lambda}-1\right) k !} \sum_{k=0}^{\infty} \sum_{q=0}^{k}(-1)^{q}\left(\begin{array}{l}
k \\
q
\end{array}\right) \int_{-\infty}^{y} x f(x) F(x)^{q} d x}{\frac{e^{\lambda}-\sum_{k=0}^{\infty} \sum_{q=0}^{k} \frac{\lambda^{k}}{k !}\left(\begin{array}{l}
k \\
q
\end{array}\right)(-1)^{q} F(x)^{q}}{e^{\lambda}-1}},
$$


where $\lambda \in(-\infty, 0) \cup(0, \infty)$, the random variable $X$ has PDF $f(x)$ and $C D F F(x)$, and $\mu$ is the mean of the Poisson- $X$ family.

\subsection{Mean Residual Life}

The mean residual life is defined as $\bar{m}(y)=\frac{1}{1-F(y)}\left[\mu-\int_{-\infty}^{y} x f(x) d x\right]-y$. For the Modified Poisson-X family of distributions, we have the following

Theorem 5.13. The mean residual life for the Modified Poisson-X family of distributions is given by

$$
\begin{gathered}
\bar{m}(y)=\left(1-\frac{e^{\lambda}-\sum_{k=0}^{\infty} \sum_{q=0}^{k} \frac{\lambda^{k}}{k !}\left(\begin{array}{l}
k \\
q
\end{array}\right)(-1)^{q} F(y)^{q}}{e^{\lambda}-1}\right)^{-1} \\
\times\left[\mu-\frac{\lambda^{k+1}}{\left(e^{\lambda}-1\right) k !} \sum_{k=0}^{\infty} \sum_{q=0}^{k}(-1)^{q}\left(\begin{array}{c}
k \\
q
\end{array}\right) \int_{-\infty}^{y} x f(x) F(x)^{q} d x\right]-y,
\end{gathered}
$$

where $\lambda \in(-\infty, 0) \cup(0, \infty)$, the random variable $X$ has PDF $f(x)$ and CDF $F(x)$, and $\mu$ is the mean of the Poisson- $X$ family.

\subsection{Order Statistics}

The pdf for the $p$ th order statistics $X_{p: n}$ of an independent identically distributed random sample, $X_{1}, X_{2}, \cdots, X_{n}$, of size $n, f_{X_{p: n}}(x)$, is given by

$$
f_{X_{p: n}}(x)=\frac{n !}{(p-1) !(n-p) !} F(x)^{p-1}(1-F(x))^{n-p} f(x)
$$

for $p=1,2, \cdots, n$.

Lemma 5.14. The $p d f$ for the pth order statistics $X_{p: n}$ of an independent identically distributed random sample, $X_{1}, X_{2}, \cdots, X_{n}$, of size $n, f_{X_{p: n}}(x)$, can be expressed as

$$
f_{X_{p: n}}(x)=\sum_{j=0}^{n-p} \omega_{j} f(x) F(x)^{p-1+j}
$$


where

$$
\omega_{j}=\frac{n !}{(p-1) !(n-p) !}\left(\begin{array}{c}
n-p \\
j
\end{array}\right)(-1)^{j} .
$$

Lemma 5.15. Let $F(x)$ be given by the CDF of the Modified Poisson-X family of distributions. Then $F(x)^{p-1+j}$ can be expressed as

$$
\sum_{v=0}^{p-1+j} \sum_{m=0}^{\infty} \omega_{v, m} F(x)^{m}
$$

where

$$
\begin{gathered}
\omega_{v, m}=\frac{e^{\lambda(p-1+j)}}{\left(e^{\lambda}-1\right)^{p-1+j}}\left(\begin{array}{c}
p-1+j \\
v
\end{array}\right) \frac{(-1)^{m+v} v^{m} \lambda^{m}}{m !} \\
\lambda \in(-\infty, 0) \cup(0, \infty)
\end{gathered}
$$

and $F(x)$ is some baseline $C D F$.

Corollary 5.16. The PDF in Theorem 5.2 can be expressed as

$$
\sum_{k=0}^{\infty} \sum_{q=0}^{k} \omega_{k, q} f(x) F(x)^{q}
$$

where

$$
\begin{gathered}
\omega_{k, q}=\frac{\lambda^{k+1}}{\left(e^{\lambda}-1\right) k !}(-1)^{q}\left(\begin{array}{l}
k \\
q
\end{array}\right) \\
\lambda \in(-\infty, 0) \cup(0, \infty)
\end{gathered}
$$

and $F(x)$ is some baseline $C D F$, and $f(x)$ is some baseline PDF.

By combining Lemma 5.14, Lemma 5.15, and Corollary 5.16, we get the following

Theorem 5.17. The $p d f, f_{X_{p: n}}(x)$, for the pth order statistics $X_{p: n}$ of an independent identically distributed random sample, $X_{1}, X_{2}, \cdots, X_{n}$, of size $n$, from the Modified Poisson-X family is given by

$$
f_{X_{p: n}}(x)=\sum_{j=0}^{n-p} \sum_{v=0}^{p-1+J} \sum_{m, k=0}^{\infty} \sum_{q=0}^{k} \omega_{j} \omega_{v, m} \omega_{k, q} f(x) F(x)^{m+q} .
$$




\subsection{Moments of Order Statistics}

The $r$ th non-central moments of the $p$ th order statistics is given by

$$
E\left(X_{p: n}^{r}\right)=\int_{-\infty}^{\infty} x^{r} f_{X_{p: n}}(x) d x
$$

For the Poisson-X family of distributions we have the following using Theorem 5.17.

Theorem 5.18. The rth noncentral moments of the pth order statistics for the Poisson-X family is given by

$$
E\left(X_{p: n}^{r}\right)=\sum_{j=0}^{n-p} \sum_{v=0}^{p-1+J} \sum_{m, k=0}^{\infty} \sum_{q=0}^{k} \omega_{j} \omega_{v, m} \omega_{k, q} \int_{-\infty}^{\infty} x^{r} f(x) F(x)^{m+q} d x .
$$

\section{Parameter Estimation}

The method of maximum likelihood is used in this paper to estimate model parameters. Here we discuss this method for the Modified Poisson-X family of distributions. Suppose $x_{1}, x_{2}, \cdots, x_{n}$ is a random sample of size $n$ from the Modified Poisson-X family of distributions. It can be shown that the total log-likelihood function is given by

$$
l=n \ln (\lambda)-n \ln \left(e^{\lambda}-1\right)+\sum_{i=1}^{n} \ln f\left(x_{i} ; \psi\right)+n \lambda-\lambda \sum_{i=1}^{n} F\left(x_{i} ; \psi\right),
$$

where $\psi$ is a vector of parameters associated with the baseline distribution. Partial differentiation of the total log-likelihood function with respect to model parameters gives the following as the score functions

$$
\begin{gathered}
\frac{\partial l}{\partial \lambda}=\frac{n}{\lambda}-\frac{e^{\lambda}}{e^{\lambda}-1}+n-\sum_{i=1}^{n} F\left(x_{i} ; \psi\right) \\
\frac{\partial l}{\partial \psi}=\sum_{i=1}^{n} \frac{\frac{\partial f\left(x_{i} ; \psi\right)}{\partial \psi}}{f\left(x_{i} ; \psi\right)}-\lambda \sum_{i=1}^{n} \frac{\partial F\left(x_{i} ; \psi\right)}{\partial \psi} .
\end{gathered}
$$


Equating the score functions to zero and numerically solving the system of equations using techniques such as the quasi Newton-Raphson method, gives the maximum likelihood estimates for the model parameters. Let $\triangle=(\lambda ; \psi)$, for the purposes of constructing confidence intervals for the parameters in the Poisson-X family of distributions, the observed information matrix, call it $J(\triangle)$, can be used due to the complex nature of the expected information matrix. The observed information matrix is given by

$$
J(\triangle)=-\left[\begin{array}{ll}
\frac{\partial^{2} \ln L}{\partial^{2} \lambda} & \frac{\partial^{2} \ln L}{\partial \lambda \partial \psi} \\
& \frac{\partial^{2} \ln L}{\partial^{2} \psi}
\end{array}\right] .
$$

The elements of the observed information matrix are given below

$$
\begin{gathered}
\frac{\partial^{2} \ln L}{\partial^{2} \lambda}=\frac{-n}{\lambda}-\frac{1}{2-2 \cosh (\lambda)} \\
\frac{\partial^{2} \ln L}{\partial \lambda \partial \psi}=-\sum_{i=1}^{n} \frac{\partial F\left(x_{i} ; \psi\right)}{\partial \psi} \\
\frac{\partial^{2} \ln L}{\partial^{2} \psi}=\frac{\left(\frac{\partial f\left(x_{i} ; \psi\right)}{\partial \psi}\right)^{2}-f\left(x_{i} ; \psi\right) \frac{\partial^{2} f\left(x_{i} ; \psi\right)}{\partial \psi}}{\left.f\left(x_{i} ; \psi\right)^{2}\right)} .
\end{gathered}
$$

When the usual regularity conditions are satisfied and that the parameters are within the interior of the parameter space, but not on the boundary, the distribution of $\sqrt{n}(\widehat{\triangle}-\triangle)$ converges to the multivariate normal distribution $N_{p+1}\left(0, I^{-1}(\triangle)\right)$, where $I(\triangle)$ is the expected information matrix, and it is assumed that $\psi=$ $\left(\psi_{1}, \cdots, \psi_{p}\right)$. The asymptotic behavior remains valid when $I(\triangle)$ is replaced by the observed information matrix evaluated at $J(\widehat{\triangle})$. The asymptotic multivariate normal distribution $N_{p+1}\left(0, J^{-1}(\widehat{\triangle})\right)$ is a very useful tool for constructing an approximate $100(1-\xi) \%$ two-sided confidence intervals for the model parameters, where $\xi$ is the significance level. 


\section{Monte Carlo Simulation Study}

In this section we show that the method of maximum likelihood is adequate in estimating the parameters in the power series family of inverted exponentiated generalized distributions. For this, a Monte Carlo simulation study is carried out to assess the performance of the estimation method in the Modified Poisson inverted exponentiated generalized distribution (MPIEG). Samples of sizes 200, 400, 500, and 700, are drawn from the MPIEG distribution, and the samples have been drawn for the following set of parameters

(a) Set I: $(\alpha, \beta, \lambda)=(8.3,20.5,-2.9)$

(b) Set II: $(\alpha, \beta, \lambda)=(20.5,8.3,2.9)$

(c) Set III: $(\alpha, \beta, \lambda)=(8.3,8.3,-2.9)$

The maximum likelihood estimators for the parameters $\alpha, \beta$, and $\lambda$ are obtained. The procedure has been repeated 400 times, and the mean and standard deviation for the estimates are computed, and the results are summarized in Tables 4-6 for each of sets I, II, and III, respectively, considered above.

Table 4: Result of simulation study for Set I.

\begin{tabular}{ccc}
\hline & \multicolumn{2}{c}{ Parameter $\lambda$} \\
\hline Sample Size & Average Estimate & Standard Deviation \\
\hline 200 & -3.061675 & 1.885471 \\
\hline 400 & -2.623562 & 1.344442 \\
\hline 500 & -2.731107 & 1.205112 \\
\hline 700 & -2.729688 & 1.010639 \\
\hline \multicolumn{3}{c}{ Parameter $\alpha$} \\
\hline Sample Size & Average Estimate & Standard Deviation \\
\hline 200 & 8.592962 & 2.127773 \\
\hline 400 & 8.875303 & 1.721993 \\
\hline 500 & 8.715073 & 1.582886 \\
\hline 700 & 8.662126 & 1.315252 \\
\hline \multicolumn{3}{c}{ Parameter $\beta$} \\
\hline Sample Size & Average Estimate & Standard Deviation \\
\hline 200 & 22.96349 & 9.664607 \\
\hline 400 & 23.41261 & 7.411761 \\
\hline 500 & 22.77176 & 6.893993 \\
\hline 700 & 22.36556 & 5.542204 \\
\hline
\end{tabular}


From the table above, we find that the simulated estimates are close to the true values of the parameters and hence the estimation method is adequate. We have also observed that the estimated standard deviation consistently decrease with increasing sample size as seen in the graphs below.

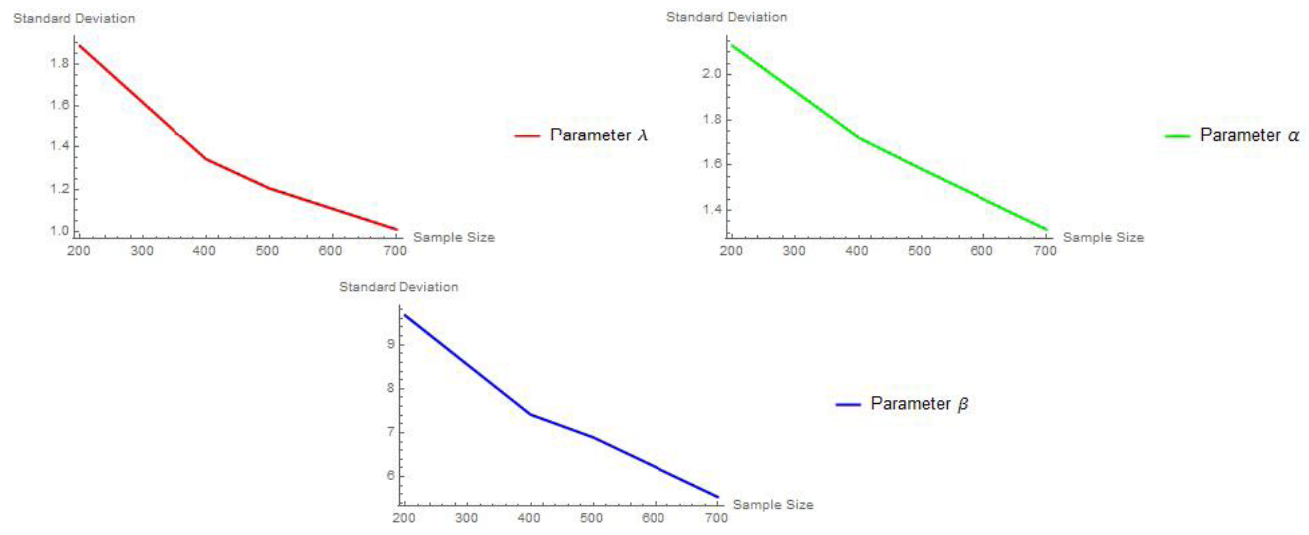

Table 5: Decreasing standard deviation for increasing sample size.

Table 6: Result of simulation study for Set II.

\begin{tabular}{ccc}
\hline & \multicolumn{2}{c}{ Parameter $\lambda$} \\
\hline Sample Size & Average Estimate & Standard Deviation \\
\hline 200 & 2.90078 & 2.045452 \\
\hline 400 & 3.0843 & 1.562558 \\
\hline 500 & 2.975228 & 1.344621 \\
\hline 700 & 3.060976 & 1.263785 \\
\hline \multicolumn{3}{c}{ Parameter $\alpha$} \\
\hline Sample Size & Average Estimate & Standard Deviation \\
\hline 200 & 20.00319 & 1.824284 \\
\hline 400 & 20.24646 & 1.194761 \\
\hline 500 & 20.27795 & 0.7811548 \\
\hline 700 & 20.33272 & 0.7474834 \\
\hline \multicolumn{2}{c}{ Parameter $\beta$} \\
\hline Sample Size & Average Estimate & Standard Deviation \\
\hline 200 & 8.954466 & 3.60614 \\
\hline 400 & 8.331257 & 2.756652 \\
\hline 500 & 8.528862 & 2.502422 \\
\hline 700 & 8.321384 & 2.327853 \\
\hline
\end{tabular}


From the table above, we find that the simulated estimates are close to the true values of the parameters and hence the estimation method is adequate. We have also observed that the estimated standard deviation consistently decrease with increasing sample size as seen in the graphs below.

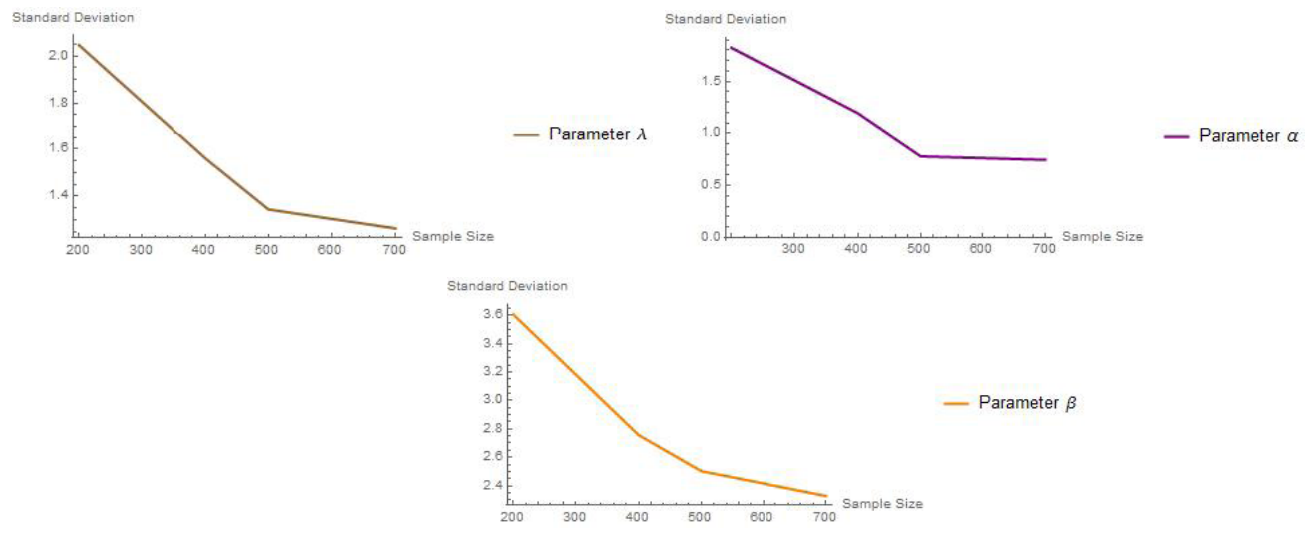

Table 7: Decreasing standard deviation for increasing sample size.

Table 8: Result of simulation study for Set III.

\begin{tabular}{ccc}
\hline & \multicolumn{2}{c}{ Parameter $\lambda$} \\
\hline Sample Size & Average Estimate & Standard Deviation \\
\hline 200 & -2.852931 & 1.944682 \\
\hline 400 & -2.690511 & 1.366186 \\
\hline 500 & -2.801686 & 1.230114 \\
\hline 700 & -2.726701 & 1.07826 \\
\hline \multicolumn{2}{c}{ Parameter $\alpha$} \\
\hline Sample Size & Average Estimate & Standard Deviation \\
\hline 200 & 8.867683 & 2.359042 \\
\hline 400 & 8.864493 & 1.877261 \\
\hline 500 & 8.68127 & 1.731915 \\
\hline 700 & 8.727292 & 1.526116 \\
\hline \multicolumn{2}{c}{ Parameter $\beta$} \\
\hline Sample Size & Average Estimate & Standard Deviation \\
\hline 200 & 8.863993 & 2.245196 \\
\hline 400 & 8.811248 & 1.671934 \\
\hline 500 & 8.682323 & 1.578819 \\
\hline 700 & 8.701399 & 1.346391 \\
\hline
\end{tabular}


From the table above, we find that the simulated estimates are close to the true values of the parameters and hence the estimation method is adequate. We have also observed that the estimated standard deviation consistently decrease with increasing sample size as seen in the graphs below.

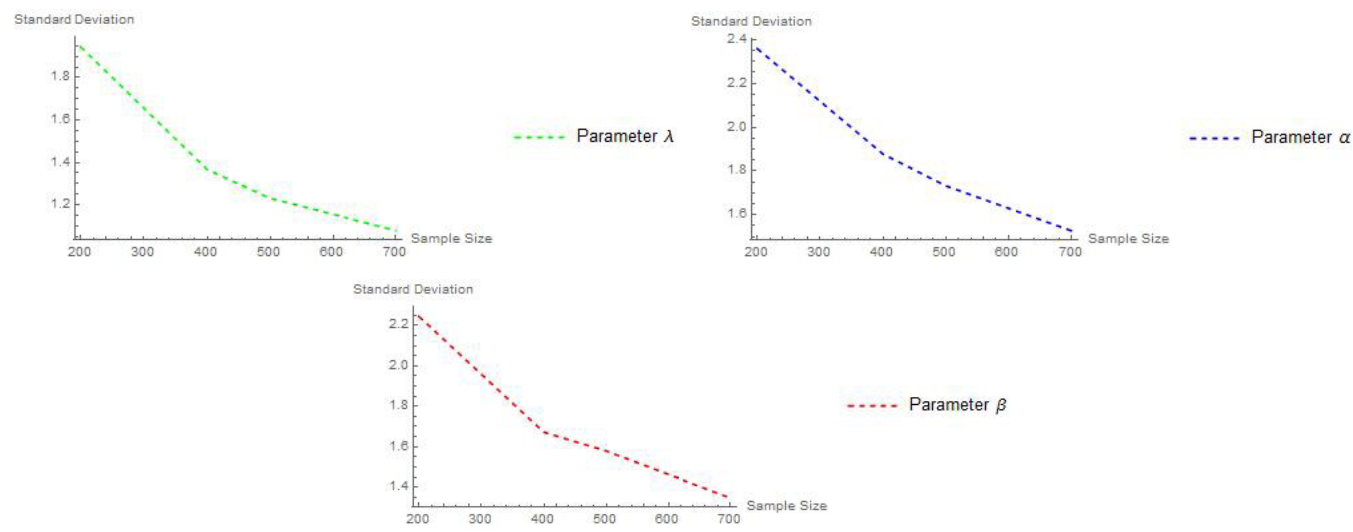

Table 9: Decreasing standard deviation for increasing sample size.

Overall the simulation study conducted, indicated that using the method of maximum likelihood in estimating model parameters is adequate.

\section{Application to Real-Life Data}

Here we demonstrate usefulness of the new family to the breaking stress of carbon fibers data, Table 2 [10]. The PDF's and CDF's of the distributions considered are already given in Section 4. We assume the modified parameter and domain space of Section 4. Using the R software, we report below in Table 10, the estimates for the parameters in each of the four distributions alongside their standard errors. 
Table 10: Estimates for the parameter of fitted distribution.

\begin{tabular}{cccc}
\hline Distribution & Parameters & Estimates & Standard error \\
\hline MPIEG & $\hat{\alpha}$ & 8.3575 & 1.7525 \\
& $\hat{\beta}$ & 20.4989 & 8.1846 \\
& $\hat{\lambda}$ & -3.0013 & 1.2541 \\
\hline MGIEG & $\hat{\alpha}$ & 5.1853 & 1.9665 \\
& $\hat{\beta}$ & 15.4842 & 5.7644 \\
& $\hat{\lambda}$ & -30.7747 & 40.2004 \\
\hline MBIEG & $\hat{\alpha}$ & 10.23557 & 1.53244 \\
& $\hat{\beta}$ & 25.79400 & 10.06697 \\
& $\hat{\lambda}$ & -0.74807 & 0.19661 \\
\hline MLIEG & $\hat{\alpha}$ & 8.9122 & 1.2876 \\
& $\hat{\beta}$ & 34.1047 & 10.0191 \\
& $\hat{\lambda}$ & -53.8299 & 74.1666 \\
\hline
\end{tabular}

The fitted CDF's to the carbon fibers data are shown below
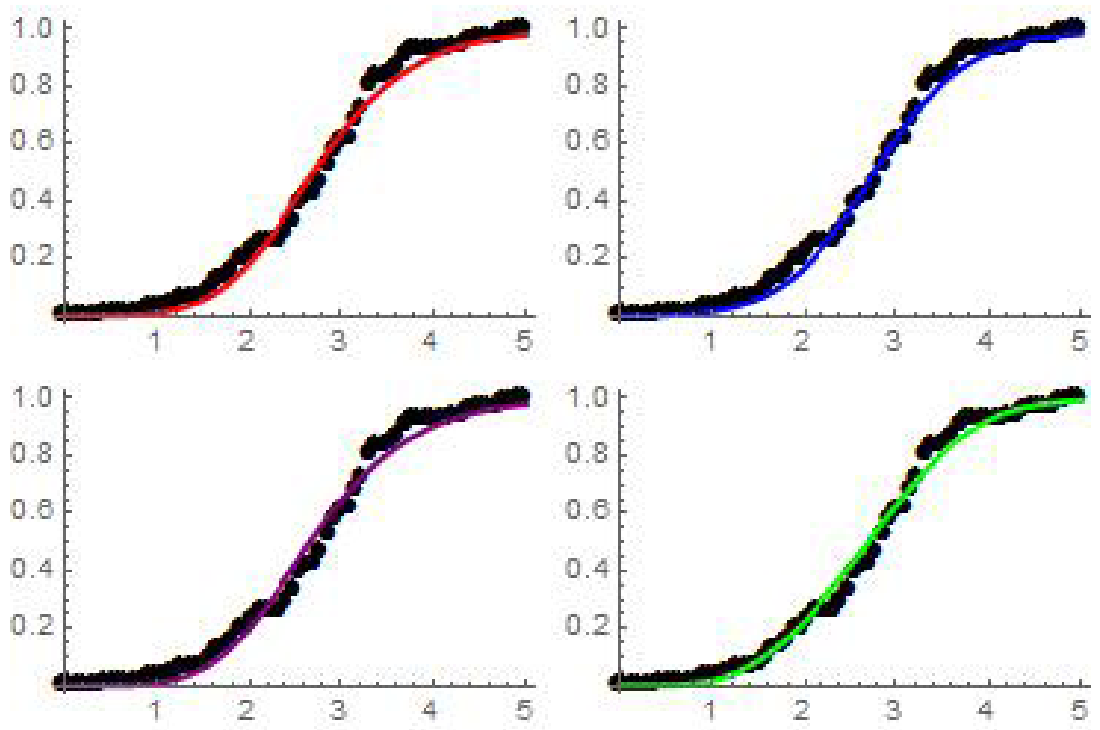

Figure 6: The fitted CDFs of MBIEG(purple), M LIEG(green), MPIEG (red), and MGIEG(blue) to the empirical distribution of Table 2 [10]. 
and the fitted PDF's are shown below
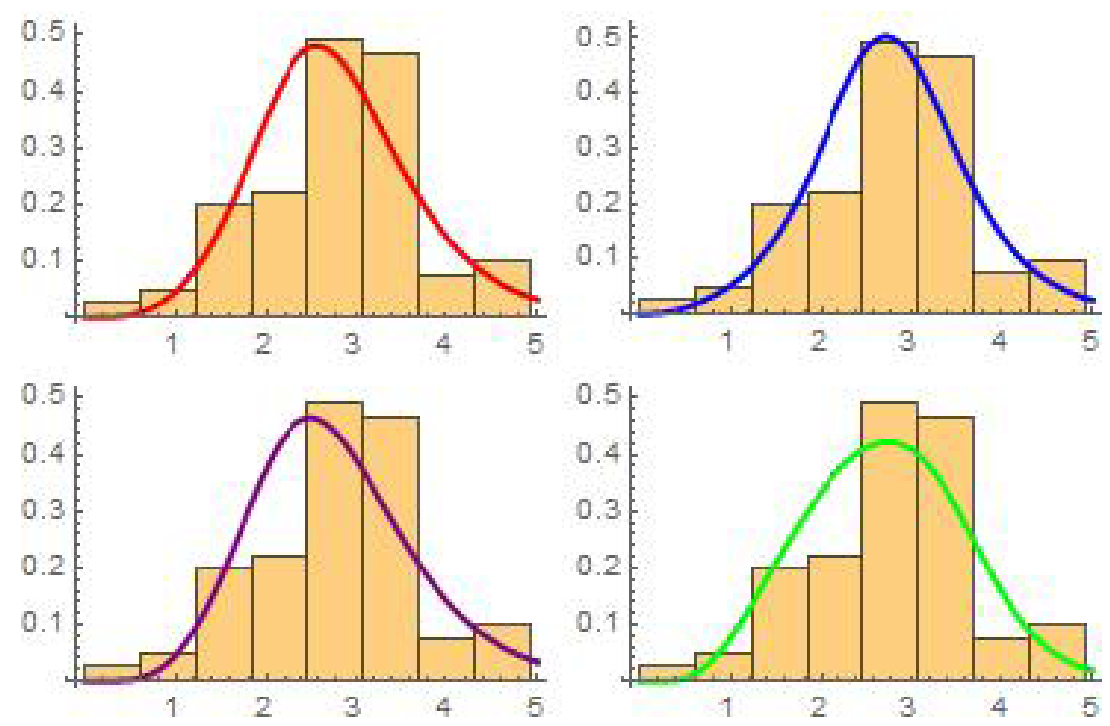

Figure 7: The fitted PDFs of MBIEG(purple), MLIEG(green), MPIEG (red), and MGIEG(blue) to the histogram of Table 2 [10].

The measures of goodness of fit we consider include Akaike information criterion (AIC), Bayesian information criterion (BIC), -2Log-Likelihood, and second-order Akaike Information Criterion (AICc) and they are reported in Table 11. Whilst it appears from the fits above, that all the distributions are competitive in fitting the breaking stress of carbon fibers data, Table 11 reveals that the MGIEG distribution is most compatible with this data set, and hence can be considered the best in this instance.

Table 11: Goodness-of-fit measures.

\begin{tabular}{ccccc}
\hline Distribution & AIC & BIC & AICc & -2Log-Likelihood \\
\hline MPIEG & 183.5427 & 190.1117 & 183.9298 & 177.5427 \\
\hline MGIEG & 178.0328 & 184.6018 & 178.4199 & 172.0328 \\
\hline MBIEG & 186.7326 & 193.3016 & 187.1197 & 180.7326 \\
\hline MLIEG & 181.4471 & 188.016 & 181.8342 & 175.4471 \\
\hline
\end{tabular}




\section{Some Characterization Theorems}

The characterization of statistical distributions plays a major role in stochastic modeling. In this section we present some characterizations of the Modified Poisson-X family of distributions. Our first characterization theorem is based on a simple relationship between two truncated moments, and for related works in this direction, the reader is referred to [11]-[16].

At first, we recall the following which will be useful in Section 9.1

Theorem 9.1. [12] Let $(\Omega, \Sigma, \mathbb{P})$ be a given probability space, and let $I=[a, b]$ be an interval for some $a<b(a=-\infty, b=\infty$ might as well be allowed). Let $X: \Omega \mapsto I$ be a continuous random variable with probability distribution function $F$, and let $q_{1}$ and $q_{2}$ be two real functions on $I$ such that

$$
\mathbb{E}\left[q_{1}(X) \mid X \geq x\right]=\mathbb{E}\left[q_{2}(X) \mid X \geq x\right] \eta(x), x \in I,
$$

is defined with some real function $\eta$. Assume that $q_{1}, q_{2} \in C^{1}(I)$, and $\eta \in C^{2}(I)$, and $F$ is twice continuously differentiable and strictly monotone increasing on the set $I$. Finally, assume that the equation $\eta q_{2}=q_{1}$ has no real solutions in the interior of $I$. Then $F$ is uniquely determined by the functions $q_{1}, q_{2}, \eta$. In particular,

$$
F(x)=\int_{a}^{x} C\left|\frac{\eta^{\prime}(u)}{\eta(u) q_{2}(u)-q_{1}(u)}\right| \exp (-s(u)) d u,
$$

where the function $s$ is a solution of the differential equation

$$
s^{\prime}=\frac{\eta^{\prime} q_{2}}{\eta q_{2}-q_{1}}
$$

and $C$ is a constant chosen to make $\int_{I} d F=1$.

Remark 9.2. The characterization based on the ratio of two truncated moments is stable in the sense of weak convergence, and for more details see [17].

In Section 9.2 we employ a single function $\psi$ of $X$ and state characterization results in terms of $\psi(X)$. At first we recall the following two results that will be useful in the sequel. 
Proposition 9.3. [18] Let $X: \Omega \mapsto(a, b)$ be a continuous random varable with CDF $F$. Let $\psi(x)$ be a differentiable function on $(a, b)$ with $\lim _{x \rightarrow a^{+}} \psi(x)=\delta>1$ and

$$
\lim _{x \rightarrow b^{-}} \psi(x)=\infty
$$

Then

$$
\mathbb{E}\left[(\psi(X))^{\delta} \mid X \leq x\right]=\delta(\psi(x))^{\delta-1}, x \in(a, b),
$$

implies

$$
\psi(x)=\delta\left[1-(F(x))^{\frac{1}{1-\delta}}\right]^{-1}, x \in(a, b) .
$$

Proposition 9.4. [18] Let $X: \Omega \mapsto(a, b)$ be a continuous random varable with $C D F$ $F$. Let $\psi_{1}(x)$ be a differentiable function on $(a, b)$ with $\lim _{x \rightarrow a^{+}} \psi_{1}(x)=\frac{\delta}{2}>\frac{1}{2}$ and

$$
\lim _{x \rightarrow b^{-}} \psi_{1}(x)=\infty
$$

Then

$$
\mathbb{E}\left[\left(\psi_{1}(X)\right)^{\delta} \mid X \leq x\right]=\delta\left(\psi_{1}(x)\right)^{\delta-1}, x \in(a, b),
$$

implies

$$
\psi_{1}(x)=\delta\left[1+(1-F(x))^{\frac{1}{1-\delta}}\right]^{-1}, x \in(a, b)
$$

\subsection{Characterization Based on Two Truncated Moments}

The main result here is the following which we state without proof

Proposition 9.5. Let $X: \Omega \mapsto \mathbb{R}$ be a continuous random variable, and let $q_{2}(x)=$ 1 and

$$
q_{1}(x)=1-\frac{e^{\lambda(1-F(x))}-1}{e^{\lambda}-1},
$$

where $\lambda \in(-\infty, 0) \cup(0, \infty)$ and $F(x)$ is some baseline CDF. The PDF of $X$ is

$$
\frac{\lambda}{e^{\lambda}-1} f(x) e^{\lambda(1-F(x))}
$$

where $f(x)$ is the PDF of the baseline distribution iff the function $\eta$ of Theorem 8.1 is given by

$$
\eta(x)=\frac{1}{2}-\frac{e^{\lambda(1-F(x))}-1}{2\left(e^{\lambda}-1\right)} .
$$


If $q_{2}$ is given by the previous Proposition, then we have the following

Corollary 9.6. Let $X: \Omega \mapsto \mathbb{R}$ be a continuous random variable. The PDF of $X$ is

$$
\frac{\lambda}{e^{\lambda}-1} f(x) e^{\lambda(1-F(x))}
$$

iff there exists functions $q_{1}$ and $\eta$ defined in Theorem 8.1 satisfying the following differential equation

$$
\frac{\eta^{\prime}(x)}{\eta(x)-q_{1}(x)}=\frac{\frac{\lambda}{e^{\lambda}-1} f(x) e^{\lambda(1-F(x))}}{1-\frac{e^{\lambda(1-F(x))}-1}{e^{\lambda}-1}} .
$$

Remark 9.7. The general solution of the above differential equation is given by

$$
\eta(x)=\left(1-\frac{e^{\lambda(1-F(x))}-1}{e^{\lambda}-1}\right)^{-1}\left[\int q_{1}(x) \frac{\lambda}{e^{\lambda}-1} f(x) e^{\lambda(1-F(x))} d x+D\right]
$$

where $D$ is a constant. One set of appropirate functions is given by the previous Proposition with $D=0$.

\subsection{Characterization Based on Conditional Expectation}

The main results here are as follows.

\subsubsection{Characterization based on Proposition 9.3}

Take $(a, b)=(0, \infty)$ and

$$
\psi(x)=\delta\left[2-\frac{e^{\lambda(1-F(x))}-1}{e^{\lambda}-1}\right]^{-1}
$$

gives a characterization of the Modified Poisson-X family of distributions. 


\subsubsection{Characterization based on Proposition 9.4}

Take $(a, b)=(0, \infty)$ and

$$
\psi_{1}(x)=\delta\left[1+\left(\frac{e^{\lambda(1-F(x))}-1}{e^{\lambda}-1}\right)^{\frac{1}{\delta-1}}\right]^{-1}
$$

gives a characterization of the Modified Poisson-X family of distributions.

\section{Further Recommendations}

The Chen distribution [19] has CDF given by

$$
G(t)=1-e^{\gamma\left(1-e^{t^{\beta}}\right)}
$$

where $\gamma, \beta, t>0$. We suggest obtaining some properties and applications of a so-called power seres inverted Chen class of distributions (using the CDF of Section 1 which is based on the stochastic representation $\left.X_{1}=\min \left\{X_{1}, \cdots, X_{n}\right\}\right)$ which is given by

$$
1-\frac{C(\lambda(1-K(x)))}{C(\lambda)},
$$

where $C$ is some useful quantity from Table 1 , and

$$
K(x)=1-G\left(\frac{1}{x+1}\right)
$$

with $G$ being the CDF of the Chen distribution.

\section{References}

[1] Godwin Dzakpasu, Power series inverted Kumaraswamy distribution with applications to lifetime data, Master Thesis in Applied Statistics, University for Development Studies, Ghana, West Africa, 2019. 
[2] A. Noack, A class of random variables with discrete distributions, Ann. Math. Statistics 21 (1950), 127-132.https://doi.org/10.1214/aoms/1177729894

[3] Gauss M. Cordeiro, Edwin M. M. Ortega and Daniel C. C. da Cunha, The exponentiated generalized class of distributions, J. Data Sci. 11 (2013), 1-27.

[4] R. C. Gupta, P. L. Gupta and R. D. Gupta, Modeling failure time data by Lehman alternatives, Comm. Statist. Theory Methods 27 (1998), 887-904. https://doi.org/10.1080/03610929808832134

[5] Wikipedia contributors, "Kumaraswamy distribution," Wikipedia, The Free Encyclopedia. 23 Jan. 2020. Web. 30 Mar. 2020

[6] Gauss M. Cordeiro and Mário de Castro, A new family of generalized distributions, $J$. Stat. Comput. Simul. 81(7) (2011), 883-898. https://doi.org/10.1080/00949650903530745

[7] Wikipedia contributors, "Inverse distribution," Wikipedia, The Free Encyclopedia. 25 Nov. 2019. Web. 15 Apr. 2020

[8] A. Rényi, On measures of entropy and information, Proceedings of the Fourth Berkeley Symposium on Mathematical Statistics and Probability 1 (1961), 547-561.

[9] Hassan Bakouch, Farrukh Jamal, Christophe Chesneau and Arslan Nasir, A new transmuted family of distributions: Properties and estimation with applications, 2017. <hal-01570370v3>

[10] Ayman Alzaatreh, Carl Lee and Felix Famoye, $T$-normal family of distributions: a new approach to generalize the normal distribution, J. Stat. Distrib. Appl. 1 (2014), 16. https://doi.org/10.1186/2195-5832-1-16

[11] W. Glänzel, A. Telcs and A. Schubert, Characterization by truncated moments and its application to Pearson-type distributions, Z. Wahrsch. Verw. Gebiete 66(2) (1984), 173-183. https://doi.org/10.1007/BF00531527

[12] W. Glänzel, A characterization theorem based on truncated moments and its application to some distribution families, Mathematical Statistics and Probability Theory, pp. 75-84, Springer, Netherlands, 1987.

https://doi.org/10.1007/978-94-009-3965-3_8 
[13] W. Glänzel, Some consequences of a characterization theorem based on truncated moments, Statistics 21(4) (1990), 613-618.

https://doi.org/10.1080/02331889008802273

[14] W. Glänzel and G. G. Hamedani, Characterization of univariate continuous distributions, Studia Sci. Math. Hungar. 37(1-2) (2001), 83-118. https://doi.org/10.1556/sscmath.37.2001.1-2.5

[15] G. G. Hamedani, Characterizations of univariate continuous distributions. II, Studia Sci. Math. Hungar. 39(3-4) (2002), 407-424.

https://doi.org/10.1556/sscmath.39.2002.3-4.11

[16] G. G. Hamedani, Characterizations of univariate continuous distributions, III, Studia Sci. Math. Hungar. 43(3) (2006), 361-385.

https://doi.org/10.1556/sscmath.43.2006.3.4

[17] Morad Alizadeh, Faton Merovci and G. G. Hamedani, Generalized transmuted family of distributions: properties and applications, Hacet. J. Math. Stat. 46(4) (2017), 645-667. https://doi.org/10.15672/HJMS .201610915478

[18] Faton Merovci, Morad Alizadeh and G. G. Hamedani, Another generalized transmuted family of distributions: properties and applications, Austrian Journal of Statistics 45 (2016), 71-93. https://doi.org/10.17713/ajs.v45i3.109

[19] Z. Chen, A new two-parameter lifetime distribution with bathtub shape or increasing failure rate function, Statist. Probab. Lett. 49 (2000), 155-161.

https://doi .org/10.1016/S0167-7152(00)00044-4

This is an open access article distributed under the terms of the Creative Commons Attribution License (http://creativecommons.org/licenses/by/4.0/), which permits unrestricted, use, distribution and reproduction in any medium, or format for any purpose, even commercially provided the work is properly cited. 